Supporting information available for JA050631Z

\title{
Theoretical Studies on the Magnetic Switching Controlled by Stacking Patterns of Bis(maleonitriledithiolato) Nickelate(III) Dimers
}

\author{
Zhaoping Ni, Xiaoming Ren, ${ }^{\dagger}$ Jing Ma, ${ }^{*}{ }^{\star}$ Jingli Xie, ${ }^{\dagger}$ Chunlin Ni, ${ }^{\dagger}$ Zhida Chen, ${ }^{\S}$ Qingjin Meng ${ }^{*}{ }^{\dagger}$
}

${ }^{\dagger}$ Coordination Chemistry Institute, State Key Laboratory of Coordination Chemistry, and ${ }^{\star}$ Institute of Theoretical and Computational Chemistry, Key Laboratory of Mesoscopic Chemistry of MOE, Nanjing University, Nanjing, 210093, P. R. China, and ${ }^{\S}$ State Key Laboratory of Rare Earth Materials Chemistry and Applications, College of Chemistry and Molecular Engineering, Peking University, Beijing, 100871, P. R. China 


\section{List of Contents:}

Table S1. Summary of intra- and intermolecular distances for 2-LT, 2-HT, and 3-HT at their experimental geometries.

Table S2. Total energies and total spin angular momentum for the singlet and triplet states, and the $J_{\mathrm{ab}}$ values using three computational schemes for 2-LT, 2-HT, and 3-HT, obtained by several DFT functional calculations with LANL2DZ basis set at their experimental geometries with Gaussian 98 program.

Table S3. The eigenvalue of the stability matrix and energy lowerings $\Delta E$ for $\mathbf{2}$-LT calculated at various levers of DFT in the singlet state.

Table S4. Total energies and total spin angular momentum for the singlet and triplet states, and the $J_{\mathrm{ab}}$ values for 2-LT, 2-HT, and 3-HT obtained by several DFT functional calculations with LANL2DZ basis set at their experimental geometries with Gaussian 03 program.

Table S5. Total energies and total spin angular momentum for the singlet and triplet states, and the $J_{\mathrm{ab}}$ values for 2-LT, 2-HT, and 3-HT obtained by UBPW91 functional calculations with several basis sets at their experimental geometries with Gaussian 98 program.

Table S6. Total energies and total spin angular momentum for the singlet and triplet states, and the $J_{\mathrm{ab}}$ values for $\mathbf{2}-\mathbf{L T}^{d}, \mathbf{2}-\mathbf{H} \mathbf{T}^{d}$, and $\mathbf{3}-\mathbf{H} \mathbf{T}^{d}$ calculated by UBPW91/LANL2DZ, with different interlayer distances $d$ on the basis of their experimental geometries with Gaussian 98 program.

Table S7. Total energies and total spin angular momentum for the singlet and triplet states, and the $J_{\mathrm{ab}}$ values for 2-LT', 2-HT', and 3-HT' calculated by UBPW91/LANL2DZ at their model structures with Gaussian 98 program.

Table S8. Total energies and total spin angular momentum for the singlet and triplet states, $J_{\mathrm{ab}}$ values, 
and $T_{\text {SOMO }}$ calculated by UBPW91/LANL2DZ with linear shifting the extent of slippage from site $K$ to $J, \Delta x$, on the basis of the model structures with Gaussian 98 program.

Table S9. Total energies and total spin angular momentum for the singlet and triplet states, and the $J_{\mathrm{ab}}$ values calculated by UBPW91/LANL2DZ with different rotation angles $\theta$ on the basis of the model structures with Gaussian 98 program.

Complete ref. 2g, 9f, 10a, c, d, 29, and 31. 
Table S1. Summary of intra- and intermolecular distances $(\AA)$ for 2-LT, 2-HT, and 3-HT at their experimental geometries.

\begin{tabular}{|c|c|c|c|c|c|c|c|c|c|}
\hline \multicolumn{10}{|c|}{ intramolecular averaged distances } \\
\hline dimer & \multicolumn{2}{|c|}{$\mathrm{Ni}-\mathrm{S}$} & \multicolumn{2}{|c|}{$\mathrm{S}-\mathrm{C}_{1}$} & \multicolumn{2}{|l|}{$\mathrm{C}_{1 \mathrm{a}}-\mathrm{C}_{1 \mathrm{~b}}$} & $\mathrm{C}_{1}-\mathrm{C}_{2}$ & \multicolumn{2}{|c|}{$\mathrm{C}_{2}-\mathrm{N}$} \\
\hline 2-LT & \multicolumn{2}{|c|}{2.149} & \multicolumn{2}{|c|}{1.717} & 1.367 & \multicolumn{2}{|c|}{1.435} & \multicolumn{2}{|c|}{1.150} \\
\hline 2-HT & \multicolumn{2}{|c|}{2.147} & \multicolumn{2}{|c|}{1.716} & 1.358 & \multicolumn{2}{|c|}{1.437} & \multicolumn{2}{|c|}{1.139} \\
\hline 3-HT & \multicolumn{2}{|c|}{2.146} & \multicolumn{2}{|c|}{1.713} & 1.364 & \multicolumn{2}{|c|}{1.437} & \multicolumn{2}{|c|}{1.140} \\
\hline \multicolumn{10}{|c|}{ intermolecular individual distances } \\
\hline dimer & Ni-Ni' & $\mathrm{S}_{\mathrm{a}}-\mathrm{S}_{\mathrm{a}}{ }^{\prime}$ & $\mathrm{S}_{\mathrm{b}}-\mathrm{S}_{\mathrm{b}}{ }^{\prime}$ & $\mathrm{S}_{\mathrm{c}}-\mathrm{S}_{\mathrm{c}}{ }^{\prime}$ & $\mathrm{S}_{\mathrm{d}}-\mathrm{S}_{\mathrm{d}}{ }^{\prime}$ & $\mathrm{Ni}-\mathrm{S}_{\mathrm{c}}^{\prime}$ & $\mathrm{Ni}-\mathrm{S}_{\mathrm{d}}^{\prime}$ & $\mathrm{Ni}^{\prime}-\mathrm{S}_{\mathrm{a}}$ & $\mathrm{Ni}^{\prime}-\mathrm{S}_{\mathrm{b}}$ \\
\hline 2-LT & 3.554 & 3.490 & 3.566 & 3.536 & 3.525 & 4.107 & 3.806 & 4.128 & 3.766 \\
\hline 2-HT & 3.950 & 3.918 & 3.865 & 3.896 & 3.995 & 4.217 & 3.786 & 4.203 & 3.812 \\
\hline 3-HT & 3.964 & 3.756 & 4.100 & 4.153 & 3.942 & 4.402 & 3.598 & 4.216 & 3.648 \\
\hline
\end{tabular}


Table S2. Total energies and total spin angular momentum for the singlet and triplet states, and the $J_{\mathrm{ab}}$ values $^{a}$ using three computational schemes $^{b}$ for 2-LT, 2-HT, and 3-HT, obtained by several DFT functional calculations ${ }^{c}$ with LANL2DZ basis set at their experimental geometries with Gaussian 98 program.

\begin{tabular}{lllllllll}
\hline dimer & method & $E_{\mathrm{BS}}$ & $\left\langle S^{2}\right\rangle_{\mathrm{BS}}$ & $E_{\mathrm{T}}$ & $<S^{2}>_{\mathrm{T}}$ & $J_{\mathrm{ab}}^{(1)}$ & $J_{\mathrm{ab}}^{(2)}$ & $J_{\mathrm{ab}}^{(3)}$ \\
\hline 2-LT & USVWN & -1461.95744974 & 0.0000 & -1461.94072971 & 2.0028 & -3669.62 & -1834.81 & -1832.25 \\
& UBP86 & -1467.55549757 & 0.0000 & -1467.54159788 & 2.0041 & -3050.63 & -1525.31 & -1522.19 \\
& UBPW91 & -1467.36384409 & 0.0000 & -1467.35044811 & 2.0045 & -2940.08 & -1470.04 & -1466.74 \\
& UBLYP & $-1465.98607601^{d}$ & 0.5441 & -1465.97787779 & 2.0188 & -1799.30 & -899.65 & -1220.11 \\
& & $(-1465.98607601)$ & $(0.5441)$ & $(-1465.97787775)$ & $(2.0188)$ & $(-1799.31)$ & $(-899.66)$ & $(-1220.12)$ \\
& UB2LYP & $-1465.53973652^{d}$ & 0.9522 & -1465.50549712 & 2.0920 & -7514.68 & -3757.34 & -6592.98 \\
& & $(-1465.53947514)$ & $(0.7777)$ & $(-1465.53325210)$ & $(2.0550)$ & $(-1365.80)$ & $(-682.90)$ & $(-1069.29)$ \\
& UB3LYP & $-1467.00488806^{d}$ & 0.4452 & -1466.99614041 & 2.0145 & -1919.89 & -959.94 & -1223.40 \\
& & $(-1467.00488805)$ & $(0.4452)$ & $(-1466.99614037)$ & $(2.0145)$ & $(-1919.89)$ & $(-959.95)$ & $(-1223.41)$ \\
& UB3PW91 & $-1466.83136661^{d}$ & 0.4674 & -1466.82283144 & 2.0144 & -1873.25 & -936.62 & -1210.89
\end{tabular}




\begin{tabular}{ccccccccc} 
UB2LYP & $-1465.53185027^{d}$ & 1.1194 & $-1465.53250661^{c}$ & 2.0439 & 144.05 & 72.02 & 155.81 \\
& $(-1465.53181326)$ & $(1.0421)$ & $(-1465.53250661)$ & $(2.0439)$ & $(152.17)$ & $(76.09)$ & $(151.90)$ \\
UB3LYP & $-1466.98823683^{d}$ & 1.0102 & -1466.98846207 & 2.0138 & 49.43 & 24.72 & 49.26 \\
& $(-1466.98823683)$ & $(1.0102)$ & $(-1466.98846212)$ & $(2.0138)$ & $(49.45)$ & $(24.72)$ & $(49.27)$ \\
UB3PW91 & $-1466.81367742^{d}$ & 1.0105 & -1466.81390012 & 2.0137 & 48.88 & 24.44 & 48.72 \\
3-HT USVWN & -1461.92711216 & 0.9775 & -1461.92722727 & 2.0028 & 25.26 & 12.63 & 24.64 \\
& & $(-1466.81367742)$ & $(1.0105)$ & $(-1466.81390017)$ & $(2.0137)$ & $(48.89)$ & $(24.44)$ & $(48.73)$ \\
UBP86 & -1467.53252577 & 0.9892 & -1467.53264387 & 2.0040 & 25.92 & 12.96 & 25.54 \\
UBPW91 & -1467.34291678 & 0.9909 & -1467.34304605 & 2.0043 & 28.37 & 14.19 & 28.00 \\
UBLYP & -1465.97592144 & 1.0129 & -1465.97627222 & 2.0180 & 76.99 & 38.49 & 79.60 \\
UB2LYP & -1465.53492516 & 1.0791 & -1465.53559760 & 2.0431 & 147.58 & 73.79 & 153.09 \\
UB3LYP & $-1466.99313707^{d}$ & 1.0085 & -1466.99343233 & 2.0139 & 64.80 & 32.40 & 64.45 \\
& $(-1466.99313725)$ & $(1.0085)$ & $(-1466.99343233)$ & $(2.0139)$ & $(64.76)$ & $(32.38)$ & $(64.41)$ \\
\hline
\end{tabular}

\footnotetext{
${ }^{a}$ Total energies are in a.u., and $J_{\mathrm{ab}}$ are in $\mathrm{cm}^{-1}$.

${ }^{b} J_{\mathrm{ab}}^{(1)}, J_{\mathrm{ab}}^{(2)}$, and $J_{\mathrm{ab}}^{(3)}$ correspond to eq 2, 3, and 4, respectively.
}

${ }^{c}$ The data listed in parentheses are obtained by using the result of UBPW91 calculation as initial guess, then calculated with other DFT functionals.

${ }^{d}$ The ALTER option is used for the singlet state calculation and all those results are checked by STABLE option. 
Table S3. The eigenvalue of the stability matrix ${ }^{a}$ and energy lowerings $\Delta E^{b}$ for 2-LT calculated at various levers of DFT in the singlet state.

\begin{tabular}{llllllll}
\hline quantity & SVWN & BP86 & BPW91 & BLYP & B2LYP & B3LYP & B3PW91 \\
\hline$\epsilon_{i}(S)$ & 0.0111 & 0.0063 & 0.0056 & -0.0110 & -0.0261 & -0.0077 & -0.0083 \\
$\Delta E$ & & & & 1.12 & 4.36 & 0.62 & 0.70
\end{tabular}

${ }^{a}$ A large negative eigenvalue implies the restricted DFT solution has a strong tendency to become an unrestricted solution.

${ }^{b}$ Energy lowings $\Delta E=E_{\mathrm{RDFT}}-E_{\mathrm{UDFT}}$ in $\mathrm{kcal} / \mathrm{mol}$. 
Table S4. Total energies and total spin angular momentum for the singlet and triplet states, and the $J_{\mathrm{ab}}$ values $^{a}$ for 2-LT, 2-HT, and 3-HT obtained by several DFT functional calculations with LANL2DZ basis set at their experimental geometries with Gaussian 03 program.

\begin{tabular}{|c|c|c|c|c|c|c|}
\hline dimer & method & $E_{\mathrm{BS}}$ & $<S^{2}>_{\mathrm{BS}}$ & $E_{\mathrm{T}}$ & $<S^{2}>_{\mathrm{T}}$ & $J_{\mathrm{ab}}$ \\
\hline \multirow[t]{7}{*}{ 2-LT } & USVWN & -1461.95744992 & 0.0000 & -1461.94072990 & 2.0028 & -1832.23 \\
\hline & UBP86 & -1467.55549788 & 0.0000 & -1467.54159820 & 2.0041 & -1522.19 \\
\hline & UBPW91 & -1467.36384436 & 0.0000 & -1467.35044838 & 2.0045 & -1466.74 \\
\hline & UBLYP & -1465.98607627 & 0.5441 & -1465.97787802 & 2.0188 & -1220.12 \\
\hline & UB2LYP & -1466.48152665 & 0.0000 & -1466.46798190 & 2.0046 & -1482.95 \\
\hline & UB3LYP & -1467.00488832 & 0.4452 & -1466.99614064 & 2.0145 & -1223.41 \\
\hline & UB3PW91 & -1466.83136684 & 0.4674 & -1466.82283165 & 2.0144 & -1210.90 \\
\hline \multirow[t]{7}{*}{ 2-HT } & USVWN & -1461.92347527 & 0.9524 & -1461.92338525 & 2.0027 & -18.81 \\
\hline & UBP86 & -1467.52582546 & 0.9799 & -1467.52580921 & 2.0040 & -3.48 \\
\hline & UBPW91 & -1467.33547935 & 0.9828 & -1467.33547892 & 2.0043 & -0.09 \\
\hline & UBLYP & -1465.97125956 & 1.0152 & -1465.97154488 & 2.0178 & 62.46 \\
\hline & UB2LYP & -1466.45450456 & 0.9815 & -1466.45449368 & 2.0045 & -2.33 \\
\hline & UB3LYP & -1466.98823714 & 1.0102 & -1466.98845243 & 2.0138 & 49.27 \\
\hline & UB3PW91 & -1466.81367770 & 1.0105 & -1466.81390044 & 2.0137 & 48.73 \\
\hline \multirow[t]{7}{*}{ 3-HT } & USVWN & -1461.92711228 & 0.9776 & -1461.92722739 & 2.0028 & 24.64 \\
\hline & UBP86 & -1467.53252594 & 0.9892 & -1467.53264404 & 2.0040 & 25.54 \\
\hline & UBPW91 & -1467.34291695 & 0.9909 & -1467.34304622 & 2.0043 & 28.00 \\
\hline & UBLYP & -1465.97592159 & 1.0129 & -1465.97627237 & 2.0180 & 76.60 \\
\hline & UB2LYP & -1466.46078776 & 0.9904 & -1466.46091214 & 2.0045 & 26.92 \\
\hline & UB3LYP & -1466.99313740 & 1.0085 & -1466.99343249 & 2.0139 & 64.42 \\
\hline & UB3PW91 & -1466.81948000 & 1.0087 & -1466.81977067 & 2.0139 & 63.46 \\
\hline
\end{tabular}

\footnotetext{
${ }^{a}$ Total energies are in a.u., and $J_{\mathrm{ab}}$ from eq 4 are shown in $\mathrm{cm}^{-1}$.
} 
Table S5. Total energies and total spin angular momentum for the singlet and triplet states, and the $J_{\mathrm{ab}}$ values $^{a}$ for 2-LT, 2-HT, and 3-HT obtained by UBPW91 functional calculations with several basis sets at their experimental geometries with Gaussian 98 program.

\begin{tabular}{|c|c|c|c|c|c|c|}
\hline dimer & basis set & $E_{\mathrm{BS}}$ & $<S^{2}>_{\mathrm{BS}}$ & $E_{\mathrm{T}}$ & $<S^{2}>_{\mathrm{T}}$ & $J_{\mathrm{ab}}$ \\
\hline \multirow{6}{*}{ 2-LT } & CEP-31G & -590.20273985 & 0.0000 & -590.19088757 & 2.0055 & -1297.07 \\
\hline & LANL2DZ & -1467.36384409 & 0.0000 & -1467.35044811 & 2.0045 & -1466.74 \\
\hline & $\mathrm{ECP} 1^{b}$ & -1467.36502919 & 0.0000 & -1467.35157978 & 2.0044 & -1472.66 \\
\hline & $\mathrm{ECP} 2^{c}$ & -4572.08740514 & 0.0000 & -4572.07404209 & 2.0048 & -1462.91 \\
\hline & SDD & -4575.06139695 & 0.0000 & -4575.05052806 & 2.0055 & -1189.45 \\
\hline & $6-311 G$ & -7250.13446175 & 0.0000 & -7250.12337450 & 2.0036 & -1214.50 \\
\hline \multirow[t]{6}{*}{ 2-HT } & CEP-31G & -590.17474906 & 0.9848 & -590.17472050 & 2.0053 & -6.14 \\
\hline & LANL2DZ & -1467.33547892 & 0.9828 & -1467.33547850 & 2.0043 & -0.09 \\
\hline & $\mathrm{ECP} 1^{b}$ & -1467.33662306 & 0.9828 & -1467.33662241 & 2.0043 & -0.14 \\
\hline & $\mathrm{ECP} 2^{c}$ & -4572.06645630 & 0.9830 & -4572.06646043 & 2.0046 & 0.89 \\
\hline & SDD & -4575.03617863 & 0.9913 & -4575.03617249 & 2.0053 & -1.33 \\
\hline & 6-311G & -7250.11750971 & 0.9840 & -7250.11749644 & 2.0035 & -2.86 \\
\hline \multirow[t]{6}{*}{ 3-HT } & CEP-31G & -590.18296180 & 0.9981 & -590.18311593 & 2.0054 & 33.58 \\
\hline & LANL2DZ & -1467.34291678 & 0.9909 & -1467.34304605 & 2.0043 & 28.00 \\
\hline & $\mathrm{ECP} 1^{b}$ & -1467.34406387 & 0.9908 & -1467.34419194 & 2.0043 & 27.73 \\
\hline & $\mathrm{ECP} 2^{c}$ & -4572.07446371 & 0.9899 & -4572.07458814 & 2.0046 & 26.91 \\
\hline & SDD & -4575.04374722 & 0.9953 & -4575.04385321 & 2.0053 & 23.03 \\
\hline & $6-311 G$ & -7250.12396822 & 0.9954 & -7250.12410245 & 2.0035 & 29.22 \\
\hline
\end{tabular}

\footnotetext{
${ }^{a}$ Total energies are in a.u., and $J_{\mathrm{ab}}$ from eq 4 are shown in $\mathrm{cm}^{-1}$.

${ }^{b}$ LANL2DZ for the complex with one set of f-polarization functions adding to the Ni atom.

${ }^{c}$ LANL2DZ for the Ni atom and the $6-31 \mathrm{G}^{*}$ basis set for $\mathrm{S}, \mathrm{C}$ and $\mathrm{N}$ atoms.
} 
Table S6. Total energies and total spin angular momentum for the singlet and triplet states, and the $J_{\mathrm{ab}}$ values for $\mathbf{2}-\mathbf{L T}^{\boldsymbol{d}}, \mathbf{2}-\mathbf{H T} \mathbf{T}^{\boldsymbol{d}}$, and $\mathbf{3}-\mathbf{H T}^{\boldsymbol{d}}$ calculated by UBPW91/LANL2DZ, with different interlayer distances $d$ on the basis of their experimental geometries with Gaussian 98 program. $^{a}$

\begin{tabular}{|c|c|c|c|c|c|c|}
\hline dimer & $d$ & $E_{\mathrm{BS}}$ & $<S^{2}>_{\mathrm{BS}}$ & $E_{\mathrm{T}}$ & $<S^{2}>_{\mathrm{T}}$ & $J_{\mathrm{ab}}$ \\
\hline \multirow[t]{8}{*}{$2-\mathbf{L T}^{d}$} & 3.00 & -1467.31681863 & 0.0000 & -1467.29479251 & 2.0054 & -2410.58 \\
\hline & 3.25 & -1467.35154460 & 0.0000 & -1467.33017902 & 2.0047 & -2339.10 \\
\hline & 3.35 & -1467.35832672 & 0.0000 & -1467.34046825 & 2.0046 & -1955.24 \\
\hline & 3.48 & -1467.36384409 & 0.0000 & -1467.35044811 & 2.0045 & -1466.74 \\
\hline & 3.55 & -1467.36523643 & 0.0000 & -1467.35345800 & 2.0044 & -1289.70 \\
\hline & 3.65 & -1467.36688812 & 0.0000 & -1467.35802548 & 2.0044 & -970.43 \\
\hline & 3.75 & $-1467.36773211^{b}$ & 0.0682 & -1467.36070525 & 2.0044 & -796.52 \\
\hline & 3.85 & $-1467.36842642^{b}$ & 0.2853 & -1467.36315674 & 2.0043 & -672.81 \\
\hline \multirow[t]{8}{*}{$2-\mathrm{HT}^{d}$} & 3.00 & -1467.27941693 & 0.9439 & -1467.27983017 & 2.0046 & 85.51 \\
\hline & 3.25 & -1467.31508381 & 0.9546 & -1467.31515228 & 2.0044 & 14.31 \\
\hline & 3.35 & -1467.32284282 & 0.9609 & -1467.32285859 & 2.0044 & 3.32 \\
\hline & 3.48 & -1467.32989201 & 0.9691 & -1467.32987382 & 2.0043 & -3.86 \\
\hline & 3.55 & -1467.33258263 & 0.9732 & -1467.33255537 & 2.0043 & -5.80 \\
\hline & 3.65 & -1467.33547892 & 0.9828 & -1467.33547850 & 2.0043 & -0.09 \\
\hline & 3.75 & -1467.33779158 & 0.9832 & -1467.33775630 & 2.0043 & -7.58 \\
\hline & 3.85 & -1467.33954744 & 0.9871 & -1467.33951419 & 2.0043 & -7.17 \\
\hline \multirow[t]{7}{*}{$3-\mathrm{HT}^{d}$} & 3.00 & -1467.30783389 & 0.8979 & -1467.30785643 & 2.0045 & 4.47 \\
\hline & 3.25 & -1467.33134518 & 0.9604 & -1467.33149704 & 2.0044 & 31.92 \\
\hline & 3.35 & -1467.33644965 & 0.9739 & -1467.33660301 & 2.0044 & 32.66 \\
\hline & 3.48 & -1467.34095642 & 0.9858 & -1467.34109716 & 2.0044 & 30.32 \\
\hline & 3.55 & -1467.34291678 & 0.9909 & -1467.34304605 & 2.0043 & 28.00 \\
\hline & 3.65 & -1467.34458598 & 0.9951 & -1467.34470059 & 2.0043 & 24.92 \\
\hline & 3.75 & -1467.34603349 & 0.9984 & -1467.34613110 & 2.0043 & 21.30 \\
\hline
\end{tabular}


$\begin{array}{llllll}3.85 & -1467.34720056 & 1.0006 & -1467.34728247 & 2.0043 & 17.91\end{array}$

${ }^{a}$ Total energies are in a.u., $J_{\mathrm{ab}}$ from eq 4 are shown in $\mathrm{cm}^{-1}$, and interlay distances $d$ are in $\AA$.

${ }^{b}$ The STABLE=OPT option is used for the singlet state calculation. 
Table S7. Total energies and total spin angular momentum for the singlet and triplet states, and the $J_{\mathrm{ab}}$ values $^{a}$ for $\mathbf{2}-\mathbf{L T}^{\prime}, \mathbf{2 - H T}{ }^{\prime}$, and 3-HT' calculated by UBPW91/LANL2DZ at their model structures with Gaussian 98 program.

\begin{tabular}{llllll}
\hline model & $E_{\mathrm{BS}}$ & $\left.<S^{2}\right\rangle_{\mathrm{BS}}$ & $E_{\mathrm{T}}$ & $\left\langle S^{2}\right\rangle_{\mathrm{T}}$ & $J_{\mathrm{ab}}$ \\
\hline 2-LT' $^{\prime}$ & -1467.34563404 & 0.0000 & -1467.33401999 & 2.0044 & -1271.70 \\
2-HT' $^{\prime}$ & -1467.34677007 & 0.9717 & -1467.34668043 & 2.0043 & -19.05 \\
3-HT' $^{\prime}$ & -1467.34644669 & 0.9948 & -1467.34661083 & 2.0043 & 35.69 \\
\hline
\end{tabular}

${ }^{a}$ Total energies are in a.u., and $J_{\mathrm{ab}}$ from eq 4 are shown in $\mathrm{cm}^{-1}$. 
Table S8. Total energies and total spin angular momentum for the singlet and triplet states, $J_{\mathrm{ab}}$ values ${ }^{a}$, and $T_{\text {SOMO }}$ calculated by UBPW91/LANL2DZ with linear shifting the extent of slippage from site $K$ to $J, \Delta x^{b}$ on the basis of the model structures with Gaussian 98 program.

\begin{tabular}{lllllll}
\hline$\Delta x$ & $E_{\mathrm{BS}}$ & $\left\langle S^{2}>_{\mathrm{BS}}\right.$ & $E_{\mathrm{T}}$ & $<S^{2}>_{\mathrm{T}}$ & $J_{\mathrm{ab}}$ & $T_{\mathrm{SOMO}}$ \\
\hline 0.00 & -1467.34469333 & 0.9664 & -1467.34460529 & 2.0043 & -18.62 & 0.19 \\
0.10 & -1467.34437304 & 0.8990 & -1467.34377375 & 2.0044 & -118.99 & 0.32 \\
0.20 & -1467.34450864 & 0.7968 & -1467.34313363 & 2.0044 & -249.90 & 0.45 \\
0.30 & -1467.34449372 & 0.6614 & -1467.34207909 & 2.0044 & -394.60 & 0.58 \\
$0.45^{c}$ & -1467.34508312 & 0.4072 & -1467.34066877 & 2.0044 & -606.59 & 0.77 \\
$0.55^{c}$ & -1467.34558382 & 0.2142 & -1467.33960457 & 2.0044 & -733.04 & 0.89 \\
$0.70^{c}$ & -1467.34651098 & 0.0002 & -1467.33801676 & 2.0044 & -930.18 & 1.00 \\
0.79 & -1467.34703543 & 0.0000 & -1467.33717778 & 2.0044 & -1079.38 & 1.00
\end{tabular}

\footnotetext{
${ }^{a}$ Total energies are in a.u., and $J_{\mathrm{ab}}$ from eq 4 are shown in $\mathrm{cm}^{-1}$.

${ }^{b} \Delta x$ are in $\AA$.

${ }^{c}$ The STABLE=OPT option is used for the singlet state calculation
} 
Table S9. Total energies and total spin angular momentum for the singlet and triplet states, and the $J_{\mathrm{ab}}$ values $^{a}$ calculated by UBPW91/LANL2DZ with different rotation angles ${ }^{b} \theta$ on the basis of the model structures with Gaussian 98 program.

\begin{tabular}{llllll}
\hline$\theta$ & $E_{\mathrm{BS}}$ & $<S^{2}>_{\mathrm{BS}}$ & $E_{\mathrm{T}}$ & $<S^{2}>_{\mathrm{T}}$ & $J_{\mathrm{ab}}$ \\
\hline 0 & -1467.34615627 & 0.9842 & -1467.34622856 & 2.0043 & 15.55 \\
4 & -1467.34619961 & 0.9876 & -1467.34629856 & 2.0043 & 21.36 \\
8 & -1467.34630605 & 0.9907 & -1467.34643259 & 2.0043 & 27.40 \\
12 & -1467.34641830 & 0.9940 & -1467.34657466 & 2.0043 & 33.97 \\
13 & -1467.34644669 & 0.9948 & -1467.34661083 & 2.0043 & 35.69 \\
16 & -1467.34657300 & 0.9974 & -1467.34676075 & 2.0043 & 40.92 \\
20 & -1467.34675770 & 1.0007 & -1467.34697541 & 2.0043 & 47.61 \\
30 & -1467.34739029 & 1.0040 & -1467.34764029 & 2.0043 & 54.85 \\
40 & -1467.34841867 & 0.9968 & -1467.34860440 & 2.0043 & 40.46 \\
50 & -1467.34955430 & 0.9916 & -1467.34966861 & 2.0043 & 24.77 \\
60 & -1467.35063899 & 1.0004 & -1467.35077043 & 2.0044 & 28.73 \\
70 & -1467.35198281 & 1.0006 & -1467.35207025 & 2.0044 & 19.12 \\
80 & -1467.35339960 & 0.9615 & -1467.35317759 & 2.0043 & -46.73 \\
90 & -1467.35443958 & 0.9133 & -1467.35386685 & 2.0043 & -115.22 \\
\hline
\end{tabular}

\footnotetext{
${ }^{a}$ Total energies are in a.u., and $J_{\mathrm{ab}}$ from eq 4 are shown in $\mathrm{cm}^{-1}$.

${ }^{b}$ Rotated angle are in degree.
} 


\section{References}

(2) (g) Sporer, C.; Ratera, I.; Ruiz-Molina, D.; Zhao, Y. X.; Vidal-Gancedo, J.; Wurst, K.; Jaitner, P.; Clays, K.; Persoons, A.; Rovira, C.; Veciana, J. Angew. Chem., Int. Ed. Engl. 2004, 43, 5266.

(9) (f) Akutagawa, T.; Shitagami, K.; Nishihara, S.; Takeda, S.; Hasegawa, T.; Nakamura, T.; Hosokoshi, Y.; Inoue, K.; Ikeuchi, S.; Miyazaki, Y.; Saito, K. J. Am. Chem. Soc. 2005, 127, 4397.

(10) (a) Rovira, C.; Veciana, J.; Ribera, E.; TarréS. J.; Canadell, E.; Rousseau, R.; Mas, M.; Molins, E.; Almeida, M.; Henriques, R. T.; Morgado, J.; Schoeffel, J. -P.; Pouget, J.-P. Angew. Chem., Int. Ed. Engl. 1997, 36, 2324. (c) Ribera, E.; Rovira, C.; Veciana, J.; Tarrés, J.; Canadell, E.; Rousseau, R.; Molins, E.; Mas, M.; Schoeffel, J. P.; Pouget, J. P.; Morgado, J.; Henriques, R. T.; Almeida, M. Chem. Eur. J. 1999, 5, 2025. (d) Ribas, X.; Mas-Torrent, M.; Pérez-Benítez, A.; Dias, J. C.; Alves, H.; Lopes, E. B.; Henriques, R. T.; Molins, E.; Santos, I. C.; Wurst, K.; Foury-Leylekian, P.; Almeida, M.; Veciana, J.; Rovira, C. Adv. Funct. Mater. 2005, 15, 1023.

(29) Frisch, M. J.; Trucks, G. W.; Schlegel, H. B.; Scuseria, G. E.; Robb, M. A.; Cheeseman, J. R.; Zakrzewski, V. G.; Montgomery, J. A., Jr.; Stratmann, R. E.; Burant, J. C.; Dapprich, S.; Millam, J. M.; Daniels, A. D.; Kudin, K. N.; Strain, M. C.; Farkas, O.; Tomasi, J.; Barone, V.; Cossi, M.; Cammi, R.; Mennucci, B.; Pomelli, C.; Adamo, C.; Clifford, S.; Ochterski, J.; Petersson, G. A.; Ayala, P. Y.; Cui, Q.; Morokuma, K.; Rega, N.; Salvador, P.; Dannenberg, J. J.; Malick, D. K.; Rabuck, A. D.; Raghavachari, K.; Foresman, J. B.; Cioslowski, J.; Ortiz, J. V.; Baboul, A. G.; Stefanov, B. B.; Liu, G.; Liashenko, A.; Piskorz, P.; Komaromi, I.; Gomperts, R.; Martin, R. L.; Fox, D. J.; Keith, T.; Al-Laham, M. A.; Peng, C. Y.; Nanayakkara, A.; Challacombe, M.; Gill, P. M. W.; Johnson, B.; Chen, W.; Wong, M. W.; Andres, J. L.; Gonzalez, C.; Head-Gordon, M.; Replogle, E. S.; Pople, J. A. Gaussian 98, revision A.11.2; Gausian, Inc., Pittsburgh, PA, 2001.

(31) Frisch, M. J.; Trucks, G. W.; Schlegel, H. B.; Scuseria, G. E.; Robb, M. A.; Cheeseman, J. R.; Montgomery, J. A., Jr.; Kudin, K. N.; Burant, J. C.; Millam, J. M.; Iyengar, S. S.; Tomasi, J.; 
Barone, V.; Mennucci, B.; Cossi, M.; Scalmani, G.; Rega, N.; Petersson, G. A.; Nakatsuji, H.; Hada, M.; Ehara, M.; Toyota, K.; Fukuda, R.; Hasegawa, J.; Ishida, M.; Nakajima, T.; Honda, Y.; Kitao, O.; Nakai, H.; Klene, M.; Li, X.; Knox, J. E.; Hratchian, H. P.; Cross, J. B.; Adamo, C.; Jaramillo, J.; Gomperts, R..; Stratmann, R. E.; Yazyev, O.; Austin, A. J.; Cammi, R.; Pomelli,C.; Ochterski, J. W.; Ayala, P. Y.; Morokuma, K.; Voth, G. A.; Salvador, P.; Dannenberg, J. J.; Zakrzewski, V. G.; Dapprich, S.; Daniels, A. D.; Strain, M. C.; Farkas, O.; Malick, D. K.; Rabuck, A. D.; Raghavachari, K.; Foresman, J. B.; Ortiz, J. V.; Cui, Q.; Baboul, A. G.; Clifford, S.; Cioslowski, J.; Stefanov, B. B.; Liu, G.; Liashenko, A.; Piskorz, P.; Komaromi, I.; Martin, R. L.; Fox, D. J.; Keith, T.; Al-Laham, M. A.; Peng, C. Y.; Nanayakkara, A.; Challacombe, M.; Gill, P. M. W.; Johnson, B.; Chen, W.; Wong, M. W.; Gonzalez, C.; Pople, J. A. Gaussian 03, revision B.03; Gausian, Inc., Pittsburgh, PA, 2003. 